\title{
Autonomic and prefrontal cortex responses to autobiographical recall of emotions
}

\author{
Carl D. Marci, Debra M. Glick, Rebecca Loh, and Darin D. Dougherty \\ Massachusetts General Hospital and Harvard Medical School, Boston, Massachusetts
}

\begin{abstract}
The present study combined measures of regional cerebral blood flow (rCBF) using positron emission tomography (PET) with measures of the autonomic nervous system using skin conductance (SC), heart rate (HR), and the high frequency band of heart rate variability (HRV) in ten healthy participants who were exposed to autobiographical scripts of memories for three target emotions: anger, happiness, and sadness. According to the results, anger was the only emotion to show a significant increase in sympathetic activity, accompanied by a significant decrease in HRV when compared with a neutral script. Anger was also the only emotion to show significant changes in $\mathrm{rCBF}$ in the prefrontal cortex. By contrast, the results for the happy and sad conditions showed no significant increase in sympathetic activity and no changes in $\mathrm{rCBF}$ in the prefrontal cortex in comparison with the neutral script. The findings suggest that a relative increase in sympathetic activity with a reciprocal decrease in parasympathetic activity may be necessary to generate frontal activity in autobiographical recall of emotions.
\end{abstract}

Researchers have made progress over the past several decades with the use of various technologies in understanding the biological basis of emotions. The typical experiment involves an emotion induction or perception task with concomitant measures of psychophysiology or neuroimaging. However, several reviews reveal the limitations of this approach in defining a uniform, biologically based pattern of response specific to even the most basic of emotions (Cacioppo, Klein, Berntson, \& Hatfield, 1993; Murphy, Nimmo-Smith, \& Lawrence, 2003). The inherent limitations of individual biologically based assessment tools may contribute to this failure. For example, whereas psychophysiology offers relatively good temporal resolution and sensitive measures of autonomic nervous system (ANS) arousal (Lang, Greenwald, Bradley, \& Hamm, 1993), there is disagreement on the extent to which physiological measures can consistently capture valence (i.e., positive vs. negative). In comparison, while neuroimaging data suggest some consensus on the structures involved in the valence of emotions (Wager, Phan, Liberzon, \& Taylor, 2003), this modality lacks a consistent index of ANS arousal or intensity of response. Thus, there is a need to combine multiple biologically based measures into one emotion research paradigm. The present study was designed to integrate both psychophysiology and neuroimaging in a context-specific emotion induction research paradigm.

The modern notion that physiology provides a basis for the experience of individual human emotions dates back to the writings of William James, who suggested that feelings follow discrete patterns of physiological response (James, 1884). Despite the popularity of this idea, nearly a century of research reveals little consistent empirical support for it. The lack of support is evidenced by a comprehensive review and meta-analyses of multiple psychophysiologic measures of the ANS that include body temperature, systemic blood pressure, respiratory rate, skin conductance (SC), and cardiac measures including heart rate (HR) that found no significant examples of emotion specific patterns of ANS activity (Cacioppo, Berntson, Klein, \& Poehlmann, 1997). The same authors conclude, in a more recent review, that despite several trends - including the finding that negative emotions, especially anger, are more arousing than positive emotions - there was wide heterogeneity in the findings and little replicable evidence for specific autonomic differences in discrete emotions (Cacioppo, Berntson, Larsen, Poehlmann, \& Ito, 2000).

Despite the focus on the ANS in the literature, few studies have separated contributions of the sympathetic and parasympathetic branches involved with emotional experiences. Frequency domain measures of cardiac function that require a mathematical transformation of the interbeat interval into power spectral densities are commonly referred to as the analyses of heart rate variability (HRV) (Akselrod et al., 1981). HRV analyses have become increasingly common because of their ability to distinguish sympathetic and parasympathetic influences on the sinoatrial pacemaker of the heart (Berntson et al., 1997). HRV has been used in a variety of emotion research studies and reduced HRV has been shown in relation to several negative emotional states, including phobic anxiety (Kawachi, Vokonas, \& Weiss, 1995), hostility (Sloan et al., 1994), and depression (Carney et al., 2000). In the present study, we include a measure of

C. D. Marci, cmarci@partners.org 
HRV to indicate the relative contributions of the parasympathetic nervous system to the target emotion.

In addition to psychophysiologic measures of the ANS, functional neuroimaging techniques as applied to the study of brain regions associated with various emotional responses have become increasingly popular over the past decade. Two recent comprehensive meta-analyses attempted to summarize the data in an effort to describe discrete neurobiological regions associated with basic emotions (Murphy et al., 2003; Phan, Wager, Taylor, \& Liberzon, 2002). The two reports included many of the same studies, and both include research with diverse emotion induction and perception techniques using functional magnetic resonance imaging (fMRI) and positron emission tomography (PET) in healthy adults. The two reviews' findings were similar to the ANS ones: little evidence for unique activation patterns for discrete emotions (e.g., happy, sad, fear, anger, and disgust). The most consistent finding in both analyses was an association between fear and activation of the amygdala, but even this finding was only found in $40 \%-60 \%$ of studies analyzed.

Whereas the results of these analyses are discouraging in their ability to confirm reproducible neurobiological correlates of discrete emotions, the analyses include a number of limitations, the most glaring of which is the failure to control for the type of emotion provocation technique used, with both analyses combining emotion induction tasks (e.g., presenting a stimulus to induce an emotion) and emotion perception tasks (e.g., viewing photos of facial expressions of emotion). Both sets of authors acknowledge the failure to control for the technique used for provoking emotions as one of several challenges that limit the ability to draw firm conclusions from these important reviews (Phan et al., 2002; Murphy et al., 2003).

As evidenced in these reviews, there are many ways of provoking emotional responses in humans. Among the most common techniques found in experimental research are the use of emotion imagery (see, e.g., Lang, Bradley, \& Cuthbert, 1998), film clips portraying emotionally evocative material (e.g., Hubert \& de Jong-Meyer, 1993), viewing of emotional facial expressions (e.g., Carr, Iacoboni, Dubeau, Mazziotta, \& Lenzi, 2003), forced expression of emotional facial expressions (e.g., Ekman, Levenson, \& Friesen, 1983), and autobiographical recall and generation of personally relevant emotional memories (e.g., Pitman, Orr, Forgue, de Jong, \& Claiborn, 1987). Each technique raises unique questions. First, is the emotion generated by predominantly internal stimuli (e.g., self-reflection on personal stories) or predominantly external stimuli (e.g., photographs depicting provocative but not personally relevant images)? Second, is the emotion that is induced personally relevant, and therefore idiosyncratic, or is it standardized? Third, how much cognitive processing is required to produce the emotion, and is this controlled for in the experiment? Fourth, is the stimulus social in nature or does it depict a scene that does not involve humans? There is evidence to suggest that all of these questions are important. For example, personal relevance has been shown to be a significant factor in the amount of physiological response induced; more personally relevant emotional scripts have resulted in more physiological arousal than have nonpersonally relevant scripts (Velasco \& Bond, 1998). In addition, the amount of cognitive processing required can mediate physiological and neuroimaging findings, resulting in suggestions to subtract neutral versions of the same stimuli from the target stimuli to control for cognitive processing (Gemar, Kapur, Segal, Brown, \& Houle, 1996).

The present study is an attempt to advance emotion research by combining data from both psychophysiology and neuroimaging measures in a research paradigm using scripts of autobiographical recall to induce specific emotions. We chose to use the recall of personally relevant emotional experiences, because previous research has found personally relevant emotion scripts to be more effective than standard emotion scripts in generating an emotional response (Velasco \& Bond, 1998). In addition, we chose to subtract the neutral script from the other emotions in an effort to control for cognitive processing used in generating the target emotion. Finally, in addition to PET imaging of the central nervous system responses, we include a measure of HRV in combination with SC, in order to monitor both the parasympathetic and sympathetic branches of the ANS, respectively (Hugdahl, 1995).

\section{METHOD}

\section{Participants}

Ten healthy participants were recruited, through advertisements in the community, as part of a larger study on emotion induction in major depressive disorder (Dougherty et al., 2004). Interested participants underwent screening for the study that included a Structured Clinical Interview for Axis I DSM-IV Disorders (First, Spitzer, Gibbon, \& Williams, 1995) in order to rule out any current or past Axis I diagnoses. In addition, participants had no history of significant neurologic or medical conditions. The participants ( 5 men and 5 women) had a mean age of $33.9(S D=11.9)$. All participants were determined to be right handed by the Edinburgh Inventory (Oldfield, 1971), and had reported normal hearing, with normal or corrected vision. Written informed consent was obtained from each participant in accordance with approval from the Human Subjects Research Committee of the Massachusetts General Hospital.

\section{Emotion Scripts}

Scripts of past emotional experiences unique to each participant were developed using a previously published procedure (Dougherty, 2004; Pitman et al., 1987). Prior to the study, participants provided written descriptions of events in their lives when they felt the most and second most of three target emotions: anger, sadness, and happiness. They also wrote two scripts describing a neutral topic (e.g., going for a walk, cooking dinner), leading to the creation of eight emotion scripts corresponding with two scripts for each emotion condition (i.e., neutrality, anger, sadness, and happiness). Using the written autobiographical scripts, an investigator in the protocol (D.D.D.) edited and composed each script into a second person narrative and, in a neutral voice, made a recording that lasted approximately $30 \mathrm{sec}$.

\section{Emotion Induction Paradigm}

Participants were scanned using a randomized block design with PET imaging. Participants were scanned a total of eight times, with two scans corresponding to the neutral condition and six scans corresponding to the target script-induced emotions. Neutral conditions were performed first and last, whereas the order of the remaining three conditions (i.e., anger, sadness, and happiness) was counterbalanced across participants. Before each scan, the participant was instructed as follows: "Close your eyes, listen carefully to the script, 
and imagine the event portrayed as vividly as possible, as if you are actually participating in the event rather than just watching yourself in it." Participants were asked to focus on the induced emotion for up to $60 \mathrm{sec}$ following the recording. Thus, during the $60 \mathrm{sec} \mathrm{im-}$ mediately after the script audiotape, participants continued to recall and imagine the event while PET data were acquired. Following termination of the PET data acquisition after the $60 \mathrm{sec}$ imaging period, participants were instructed to stop imagining the event. Emotion inductions were separated by at least $10 \mathrm{~min}$ to allow for return to resting physiologic states (defined as within $10 \%$ of baseline levels) and for radiation decay to achieve negligible levels before beginning the next emotion induction.

\section{Emotional State Subjective Report}

Immediately following the last script, participants were asked to recall and rate their subjective emotional responses to each script. Participants rated their emotional responses on separate subjective 0 - to 10 -point visual analog scales, with 0 indicating the complete absence of a response and 10 indicating the maximum possible response for the target emotions. ANOVAs were used to compare differences between ratings within subjects.

\section{Psychophysiologic Assessment}

Psychophysiologic measures were recorded continuously throughout the study period for each emotion induction. Measures included SC, HR, and derived HRV. SC and HR data were collected and analyzed using an ADInstruments PowerLab 8SP computerbased modular instrument system and Chart software Version 4.2 (ADInstruments, Inc., Sydney). The PowerLab system uses 32-bit processing at $16 \mathrm{MHz}$ with a 16-bit analog to digital converter set at a $100 \mathrm{~Hz}$ sample rate. For this study, $\mathrm{Ag}-\mathrm{AgCl}$ electrodes were used to measure SC level from the distal palmer surface of the third and fourth digits of the individual's left hand. SC values are reported in microsiemens $(\mu \mathrm{S})$. HR, including HRV variables, was extracted from the single lead chest EKG. HR is reported in interbeat intervals $(\mathrm{NN})$ in milliseconds $(\mathrm{msec})$. The HRV measurements were calculated by means of frequency domain indices using commercially available software (Chart HRV Module Version 5.0, ADInstruments Inc., Sydney, Australia). The frequency domain components computed were high-frequency power (HF: $0.15-0.40 \mathrm{~Hz}$ ), low-frequency power (LF: $0.04-0.15 \mathrm{~Hz}$ ), and the LF-HF ratio. HF and LF results are reported in $\mathrm{msec}^{2}$.

Analyses of psychophysiologic data included the $30 \mathrm{sec}$ before the reading of the script (baseline period) and the $60 \mathrm{sec}$ following the playback of the emotion script while each PET image was recorded (imaging period). SC values were obtained by computing the area under the curve (AUC), whereas HR and HRV values were averaged for each scan within the baseline and imaging periods. For each target emotion, we report paired $t$ tests for the change from baseline to imaging period, corrected for the duration of SC, HR, and the HF variable of HRV used in the analyses. We limited our HRV analyses to the HF region, because the script imaging period was only $60 \mathrm{sec}$ in duration, which is below the minimum suggested for analyses of LF regions of the power spectrum (Berntson et al., 1997). Change scores were calculated by subtracting the relevant immediate physiologic baseline from the imaging period for the target emotion and then averaging the two trials for each of the angry, sad, and happy emotion scripts. Because of the high variability in the study conditions for participants leading up to the start of the protocol-as reflected in the high variability in baseline autonomic physiology prior to the first neutral condition ( $S D$ s significantly greater than $M \mathrm{~s}$ when compared with subsequent conditions) - we limited the analyses of emotion responses to subtraction of the second neutral script occurring at the very end of the protocol. Thus, a change score was calculated for the second neutral emotion script for each participant for the purposes of comparisons to the neutral condition, as described below. In addition, square root transformations were calculated on all physiologic means to reduce variability between participants that is common in physiologic signals (Hugdahl, 1995).
A final calculation was made in order to directly compare the physiology data with the neuroimaging data and to control for effects of cognitive processing of the emotion scripts. This comparison involved independent calculations of the difference between the angry, happy, and sad change scores and the neutral script for each emotion. We report the change scores (imaging minus baseline period) for each physiologic parameter for the individual emotion conditions for the group, followed by the difference between those change scores for the target emotion script minus the neutral script.

\section{PET Imaging Assessment}

PET imaging involved a 15-slice whole-body tomograph used in its stationary mode (Scanditronix Model PC4096; General Electric Medical Systems, Milwaukee, WI). The slice geometry consisted of contiguous slices with center-to-center distance of $6.5 \mathrm{~mm}$ (axial field equal to $97.5 \mathrm{~mm}$ ) and axial resolution of $6.0-\mathrm{mm}$ full width at half maximum. Image reconstruction was performed using a computed attenuation correction and a Hanning-weighted reconstruction filter set to yield 8.0-mm in-plane spatial resolution full width at half maximum. Additional processing was performed to account for scattered radiation, random coincidence, and counting losses that were due to dead time in the camera electronics. Head alignment was made relative to the canthomeatal line using projected laser lines whose positions were known with respect to the slice positions of the scanner. An individually molded thermoplastic mask was used to minimize head motion. Once the head was in place, the participant was fitted with a pair of nasal cannulae and an overlying face mask, which were attached to the radio-labeled gas inflow and vacuum, respectively.

Immediately following each emotion induction, participants underwent PET imaging while continuously inhaling tracer quantities of ${ }^{15} \mathrm{O}$-carbon dioxide that was mixed with room air for $60 \mathrm{sec}$. The concentration of the delivered gas was $2,960 \mathrm{Mbq} / \mathrm{L}(80 \mathrm{mCi} / \mathrm{L})$, with a flow rate of $2 \mathrm{~L} / \mathrm{min}$, further diluted by the free mixture with room air within the face mask, which resulted in a rapidly rising count rate in the brain, reaching terminal count rates of 100,000 to 200,000 events/sec. Previous unpublished data at Massachusetts General Hospital using radial artery cannulation has demonstrated that the integrated counts over inhalation periods up to $90 \mathrm{sec}$ are a linear function over the flow range of $0-130 \mathrm{~mL} / \mathrm{min}$ per $100 \mathrm{~g}$. Therefore, for data to be produced with units of flow relative to the whole brain, no arterial access was necessary.

Statistical analysis of the PET data followed the theory of statistical parametric mapping (Friston, Frith, Liddle, \& Frackowiak, 1991; Friston et al., 1995). Data were analyzed using the SPM99 statistical software package (Wellcome Department of Cognitive Neurology, London). PET images were motion corrected, spatially normalized to the standard normalized space established by the Montreal Neurological Institute (available at www.bic.mni.mcgill.ca), and smoothed to $10-\mathrm{mm}$ full width at half maximum. At each voxel, the PET data were normalized by the global mean and fit to a linear statistical model by the method of least squares. Planned contrasts at each voxel were conducted; this method fits a linear statistical modelvoxel by voxel - to the data, and hypotheses were tested as contrasts in which linear compounds of the model parameters were evaluated using $t$ statistics that were then transformed to $z$ scores. We report regions containing foci of activation with $z$ scores $\geq 3.09$ (corresponding to $p \leq .001$, uncorrected for multiple comparisons). Note that the data were inspected in a hierarchical manner: First, regions from the a priori hypotheses were inspected; next, the entire brain volume was inspected and, finally, post hoc findings were reported using a comparable threshold to reduce bias.

\section{RESULTS}

\section{Emotional State Subjective Report}

Because of the constraints of the PET data acquisition, participants rated their experience of the scripts retrospectively on the emotional state scales at the conclusion 
Table 1

Differences Between Change Scores for Target Emotion Versus Neutral Autobiographical Scripts

\begin{tabular}{lrrrc}
\hline \multicolumn{1}{c}{ Emotion } & \multicolumn{1}{c}{$M$} & $S D$ & $t$ Value & $p$ Value \\
\hline Anger & & & & \\
SC AUC $(\mu \mathrm{S})$ & 4.33 & 4.20 & 3.26 & $<.01$ \\
NN interval $\left(\mathrm{msec}^{2}\right)$ & -8.17 & 4.65 & -5.55 & $<.01$ \\
HF power $\left(\mathrm{msec}^{2}\right)$ & -39.57 & 35.14 & -3.56 & $<.01$ \\
Happiness & & & & \\
SC AUC $(\mu \mathrm{S})$ & -3.99 & 11.54 & -1.09 & n.s. \\
NN interval $\left(\mathrm{msec}^{2}\right)$ & -6.57 & 5.53 & -3.76 & $<.01$ \\
HF power $\left(\mathrm{msec}^{2}\right)$ & -28.43 & 45.24 & -1.99 & n.s. \\
Sadness & & & & \\
SC AUC $(\mu \mathrm{S})$ & -1.05 & 10.45 & -0.32 & n.s. \\
NN interval $\left(\mathrm{msec}^{2}\right)$ & -4.16 & 5.93 & -2.22 & n.s. \\
HF power $\left(\mathrm{msec}^{2}\right)$ & -32.14 & 39.18 & -2.59 & $<.05$ \\
\hline
\end{tabular}

Note-AUC, area under the curve; $\mu \mathrm{S}$, microsiemens; $\mathrm{NN}$, heart rate interbeat interval; HF, high frequency.

of the final neutral emotion induction. In general, participants reported high ratings of the target emotion. The ANOVAs comparing anger, happiness, and sadness during the angry $(F=29.92)$, happy $(F=322.30)$, and sad $(F=54.47)$ emotion inductions were all significant $(p<$ $.001)$. Tukey's post hoc tests revealed that in response to the angry scripts, participants reported feeling significantly more anger than either happiness or sadness, and significantly more sadness than happiness $(p<.01)$. In response to the happy scripts, participants reported feeling significantly more happiness than either anger or sadness $(p<.001)$, with no difference between ratings of anger and sadness during the happy script. Finally, in response to the sad scripts, participants reported feeling significantly more sadness than either anger or happiness, and more anger than happiness $(p<.001)$.

\section{Psychophysiology}

The results of the psychophysiologic data are presented first as the difference between the imaging period minus the emotion-specific baseline period, and then as the difference between the target emotion and the neutral emotion condition. The difference between the target emotion and the neutral emotion for $\mathrm{SC}, \mathrm{NN}$ interval, and $\mathrm{HF}$ power are also presented in Table 1 .

\section{Neutral Condition}

There was a significant increase in SC during the neutral imaging period compared with the baseline period, with a mean increase of $3.21 \pm 3.44 \mu \mathrm{S}(t=2.95, p=$ $.016)$. In addition, HR also significantly increased from baseline to imaging period for the neutral script, with an average change in NN interval of $-0.51 \pm 0.55 \mathrm{msec}(t=$ $-2.96, p=.016)$. There were no significant changes in the HF variable of HRV during the neutral condition from baseline $(t=1.26, p=.239)$.

\section{Angry Condition}

There was a significant increase in SC during the anger imaging period compared with the baseline period, with a mean increase of $5.44 \pm 7.27 \mu \mathrm{S}(t=2.37, p=.042)$. HR also significantly increased from baseline to imaging for the angry emotion condition, with an average $\mathrm{NN}$ change of $-1.40 \pm 1.14 \mathrm{msec}(t=-3.88, p=.004)$. In addition, the $\mathrm{HF}$ variable of HRV significantly decreased during the same anger condition, with a mean decrease of $-39.57 \pm$ $35.14(t=-3.56, p=.012)$.

When compared with the neutral condition, there was a significant increase in $\mathrm{SC}$ for the anger condition, with a mean increase of $4.33 \pm 4.20 \mu \mathrm{S}(t=3.26, p=.009)$. HR also increased during the anger condition when compared with the neutral script, with a mean NN interval change of $-8.17 \pm 4.65 \mathrm{msec}(t=-5.55, p=.001)$. Finally, the $\mathrm{HF}$ variable of $\mathrm{HRV}$ also changed significantly compared with the neutral script, with a mean decrease of $-39.57 \pm$ $35.14(t=-3.56, p=.006)$.

\section{Happy Condition}

There was no significant change in SC during the happy imaging condition when compared with the baseline $(t=$ $0.82, p=.431)$. However, there was a significant increase in HR from baseline to imagery for the happy condition, with an average NN change of $-0.99 \pm .98 \mathrm{msec}(t=-3.20$, $p=.011)$. The HF variable of HRV did not significantly change from baseline to imagery $(t=-1.38, p=.202)$.

When compared with the change in SC during the neutral emotion condition, there was no significant difference in SC for the happy condition $(t=-1.09, p=$ .303). However, when compared with the change in HR during the neutral condition, there was a significant increase in HR during the happy condition with a mean NN interval change of $-6.57 \pm 5.53 \mathrm{msec}(t=-3.76, p=$ $.005)$. Although not significant, there was a trend for HF to decrease during the happy emotion script as compared with the neutral script $(t=-1.99, p=.078)$.

\section{Sad Condition}

There was no significant change in SC between the baseline and the imaging periods for the sad emotion induction condition $(t=0.53, p=.611)$. There was a trend for HR to increase from baseline to imagery during the sad emotion induction condition $(t=-2.07, p=.069)$, whereas the HF variable of HRV showed no change $(t=$ $-1.63, p=.137)$.

There was no change in SC during the sad condition when compared with the neutral condition $(t=-0.32$, $p=.757)$. There was a trend for an increase in HR during the sad condition in comparison with the neutral condition $(t=-2.22, p=.054)$. Finally, when compared with the change in the HF variable of HRV during the neutral emotion script, there was a significant decrease in the HF variable of HRV during the sad condition, with a mean decrease of $-32.14 \pm 39.18 \mathrm{msec}(t=-2.59, p=.029)$.

\section{PET Imaging}

Table 2 summarizes the areas of increased $\mathrm{rCBF}$ for each of the target emotions. Whole-brain analyses were conducted similarly to those of the psychophysiology data by independently averaging the two emotion conditions for the three target emotions and subtracting the neutral emotion. The anger-neutral comparison demonstrated increased $\mathrm{rCBF}$ in the left orbital frontal cortex (OFC; $z=$ 
Table 2

Significant Differences in Increased Cerebral Blood Flow in Specific Brain Regions During Target Emotion Induction Versus Neutral Autobiographical Script

\begin{tabular}{lccc}
\multicolumn{4}{c}{ Neutral Autobiographical Script } \\
\multicolumn{1}{c}{ Brain Region } & & & $\begin{array}{c}\text { MNI Coordinates } \\
(x, y, z)\end{array}$ \\
\hline Anger - Neutral & Cluster Size & $z$ Score & \\
$\quad$ Orbital frontal cortex & 49 & 3.42 & $-10,66,-12$ \\
$\quad$ Superior temporal gyrus & 215 & 3.48 & $50,-24,2$ \\
Insula & 63 & 3.41 & $-28,-18,12$ \\
Cerebellum & 27 & 3.30 & $-46,-72,-30$ \\
Middle temporal gyrus & 41 & 3.26 & $-58,-44,8$ \\
Sad - Neutral & & & \\
Cuneus & 191 & 4.09 & $-2,-76,8$ \\
Caudate & 321 & 3.73 & $-14,18,10$ \\
$\quad$ Middle temporal gyrus & 55 & 3.13 & $-58,-44,6$ \\
Happy - Neutral & & & \\
$\quad$ Ventral striatum & 137 & 3.58 & $-18,26,0$ \\
Middle temporal gyrus & 155 & 3.89 & $-56,-44,6$ \\
Anterior temporal pole & 13 & 3.21 & $-38,24,-34$ \\
Superior temporal gyrus & 21 & 3.12 & $42,-36,14$ \\
\hline
\end{tabular}

3.42; MNI coordinates $=-10,66,-12$ ), as well as in the left insula $(z=3.41$; MNI coordinates $=-28,-18$, $12)$, the left cerebellum $(z=3.30$; MNI coordinates $=$ $-46,-72,-30)$, the right superior temporal gyrus $(z=$ 3.48; MNI coordinates $=50,-24,2)$, and the left middle temporal gyrus $(z=3.26$; MNI coordinates $=-58$, $-44,8)$. The happy-neutral comparison demonstrated no significant increases in the prefrontal cortex, but significantly increased $\mathrm{rCBF}$ in the left ventral striatum $(z=$ 3.58; MNI coordinates $=-18,26,0)$, the left anterior temporal pole $(z=3.21$; MNI coordinates $=-38,24$, $-34)$, the right superior temporal gyrus $(z=3.12$; MNI coordinates $=42,-36,14)$, and the left middle temporal gyrus $(z=3.89$; MNI coordinates $=-56,-44,6)$. Finally, the sad-neutral comparison also did not demon- strate any significant changes in $\mathrm{rCBF}$ in the prefrontal cortex. However, the sad-neutral comparison did reveal significant rCBF increases in the left cuneus $(z=4.09$; MNI coordinates $=-2,-76,8)$, the left caudate $(z=$ 3.73; MNI coordinates $=-14,18,10$ ), and the left middle temporal gyrus $(z=3.13$; MNI coordinates $=-58$, $-44,6)$.

Figure 1 illustrates changes in PET imaging activity, as well as the concomitant changes in psychophysiology for anger. Note that the results for the HRV have been transformed for Figure 1 by dividing by a constant $(k=10)$ in order for the data to fit on the same graph.

\section{DISCUSSION}

The present study combined measures of activity in the central nervous system using PET imaging with measures of activity in the peripheral nervous system using SC, HR, and HRV. The analyses of these multiple measures in healthy participants involved recall of autobiographical memories for three target emotions: anger, happiness, and sadness. The results show that anger was the only target emotion to show an increase in sympathetic nervous system activity when compared with the neutral condition. This increase in sympathetic activity was accompanied by a significant reciprocal decrease in parasympathetic activity, as reflected in decreased HF band of HRV and increased HR. Interestingly, anger was the only emotion to show significant activity in the prefrontal cortex, with significant increases in rCBF in the left OFC. In comparison, the results for the happy and sad conditions showed no significant increase in sympathetic activity and no activity in the frontal cortex compared with the neutral condition, despite decreases in parasympathetic activity. The findings suggest that increased sympathetic activity with reciprocal decreased
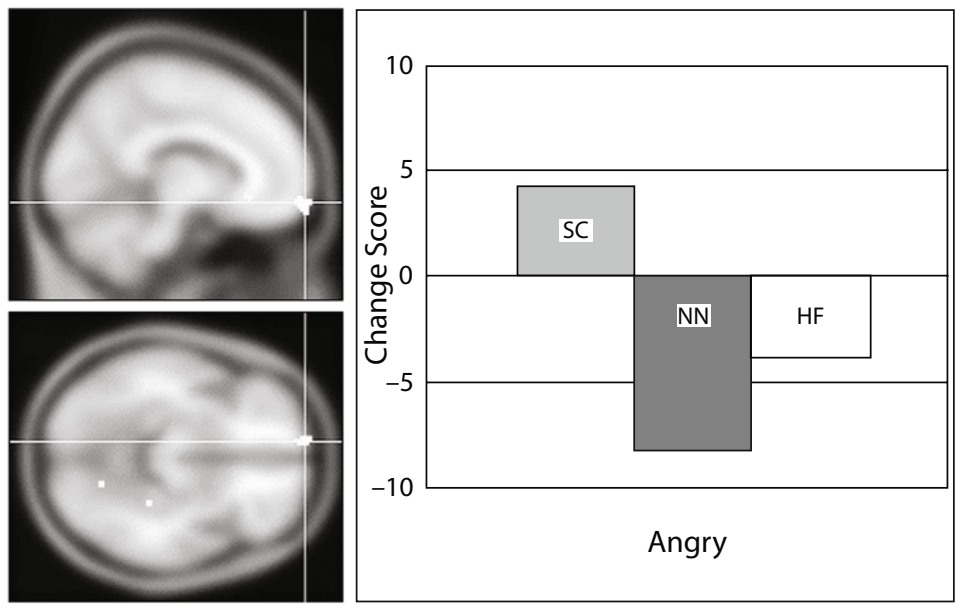

Figure 1. Significant physiologic responses and corresponding areas of increased $\mathrm{rCBF}$ on PET imaging for emotion induction of anger using autobiographical recall. Change scores represent the differences between the period of emotion induction and relative baseline. For the HF variable, absolute change was transformed to fit the graph.All change scores are significant changes from neutral $(p<.01)$. SC, skin conductivity; NN, heart rate interbeat interval; HF, high frequency heart rate variability. 
parasympathetic activity may be necessary to generate frontal activity in autobiographical recall of emotions.

Most theories of emotion support an essential role for physiology and neurobiology (Ekman \& Davidson, 1994). Despite this fact, few studies have combined measures of both factors in research paradigms designed to study emotion. Thus, to date, it has been difficult to assess the role of autonomic activity in neuroimaging studies and similarly difficult to assess central nervous system responses in studies that rely exclusively on physiology. However, one consistency in the physiology literature is the finding that negative emotions tend to have a steeper arousal curve when compared with positive emotions (Bradley, 2000). In the present study, the low peripheral arousal of sadness and happiness - as evidenced by the decrease in $\mathrm{SC}$ activity in response to the relevant autobiographical memories compared with the neutral emotion-had different central activity (cuneus vs. striatum, respectively) and no significant activity in the prefrontal cortex. In contrast, the anger induction generated high levels of arousal, as evidenced by the reciprocal increase in sympathetic (SC) and decrease in parasympathetic (HF) activity. This high arousal was associated with increased left OFC activity during the anger condition. This anger finding supports previous studies that show high arousal (Christie \& Friedman, 2004) and left frontal activity (Dougherty et al., 2004; Goldstein et al., 2005) during anger emotion induction. Although speculative, these findings suggest a new model of emotion that requires a minimal threshold level of arousal to induce a response in the relevant region of the prefrontal cortex.

The present study also supports the need to consider the relative characteristics of the emotion stimulus and the ability of a multidisciplined approach for elucidation of the unique features of autobiographical recall of emotion. For example, the comparison of the baseline to imagery period for the neutral stimulus suggests that perhaps "neutral" autobiographical scripts are not so neutral. The findings of a significant increase in SC and HR without a significant increase in the HF band of HRV suggest increased sympathetic activity in healthy participants to the neutral autobiographical script. This finding is particularly noteworthy, given the fact that only the second neutral script was used, thus minimizing idiosyncratic factors such as the varying levels of state anxiety prior to the start of the research protocol. It is possible that this increased sympathetic activity represents cognitive processing, which may include an orienting response to and language processing of the neutral verbal script, a hypothesis that is worthy of future study.

Another finding related to stimulus type is the lack of activity in the amygdala for any of the target emotions in the present study. Several studies now support the finding of amygdala activity for both positive and negative emotions (Kensinger \& Schacter, 2006; Wager et al., 2003). In addition, there are now several studies that support the role of the amygdala in emotion response beyond a simple reaction to fear-related stimuli (Glascher \& Adolphs, 2003; Wright et al., 2000). Consistent with Phan et al.'s (2002) proposal that the amygdala may respond to any personally meaningful stimuli, it is possible that in the present study, the neutral autobiographical scripts were quite meaningful to participants (since they were based on personal experience), thus eliminating any difference in the amygdala activation between the target emotion and the neutral condition. Because we did not scan during a nonscript neutral condition, we cannot be sure that neutral is not at least partially affectively loaded due to the personally relevant nature of the stimulus, although the participant self-reports argue against this interpretation. An alternative explanation - given the temporal dynamics of PET rCBF - may involve the timing of amygdalar responses to nonthreatening stimuli as transient and nonsignificant in the context of a full minute of recording, as used in this research protocol. Regardless of the reasons, further consideration of what is "neutral" in emotion induction and future studies examining other types of neutral scripts are warranted.

Another finding of note in the present study is the common area of activation in PET activity that may be related to the type of induction technique used. In the present study, the only region of activity on PET imaging that was common to all three target emotions was the left middle temporal gyrus. Given that this area was not commonly active in the two meta-analyses discussed in the introduction (Murphy et al., 2003; Phan et al., 2002), it is possible that this area is unique to emotions induced by verbal playback of autobiographical scripts. This finding is supported by a recent neuroimaging study in amnesic patients (Buchanan, Tranel, \& Adolphs, 2005). The authors found that when comparing patients with discrete versus extensive hippocampal damage and normal controls, patients with more extensive damage to the surrounding temporal cortex had significantly reduced quality of autobiographical memories for unpleasant events. Given this finding and the emerging relationship between decreased volume of the left middle temporal gyrus and increased auditory hallucinations in patients with chronic schizophrenia (Onitsuka et al., 2004) — one can speculate that the left middle temporal gyrus findings in the present study are related to the verbal and auditory nature of the emotion-induction scripts. At the very least, more careful consideration of the induction technique used and controls for language versus image-based approaches in emotion research is warranted.

The present study has several important limitations. First, despite attempts to collect subjective ratings of the target emotion, these ratings were given retrospectively following completion of all the emotion induction scripts and associated imaging. In addition, the ratings do not include a subjective report of level of arousal, an important consideration in the interpretation of emotional responses. Future studies should consider giving subjective recall questionnaires immediately following the experience and should include ratings of arousal. Second, as noted previously, the present study relied on autobiographical scripts to generate target emotions. Future studies should carefully consider the neutral condition and combine multiple induction tasks into a single research paradigm so that factors such as cognitive loading, personal relevance, 
the social nature of the stimulus, and approach-versusavoidance parameters can be controlled for and evaluated more thoroughly. Third, given the differences in the temporal dynamics of PET and peripheral measures of autonomic physiology, correlation and other statistical analyses combining all data types in this study are difficult. Future studies using fMRI and physiologic parameters may overcome this limitation and provide an opportunity for corresponding network type analyses. Finally, in the present study, little consideration of the cognitive-emotional style of the participants was made. There is evidence to support a distinction between arousal- and valence-focused individuals, which may bias subjective ratings of emotion (Barrett, 2004; Barrett \& Niedenthal, 2004). Future studies that control for cognitive-emotional style while collecting measures of central and peripheral nervous system activity are needed.

Despite these limitations, the present study successfully combined neuroimaging and measures of both divisions of the ANS in an emotion-induction paradigm. One possible explanation for the heterogeneity of findings in recent reviews of both physiology and neuroimaging in the emotion literature is the association in the brain of differential networks representing discrete emotions with similar downstream physiologic responses that depend on both the level of arousal and the valence of the particular emotion. The present findings support a multidisciplinary approach, combining central and peripheral measures in emotion research. They suggest that future research studies including multiple measures and controlling for multiple variables - including type of emotion inductionare needed to further evaluate the age-old question of the unique nature of emotions and the disorders associated with them.

\section{AUTHOR NOTE}

Funding for this project for C.D.M. comes from NIMH Grant F32MH072073. Thanks for financial support also go to the MGH Endowment for the Advancement of Psychotherapy and to Constance and Stephen Lieber in support of the National Alliance for Research on Schizophrenia and Depression. Funding for D.D.D. comes from NIMH Grant K23MH001735. Address correspondence to C. D. Marci, M.D., Director of Social Neuroscience, Massachusetts General Hospital, Department of Psychiatry, 15 Parkman St., WACC 812, Boston, MA 02114 (e-mail: cmarci@partners.org).

\section{REFERENCES}

Akselrod, S., Gordon, D., Ubel, F. A., Shannon, D. C., Berger, A. C., \& Cohen, R. J. (1981). Power spectrum analysis of heart rate fluctuation: A quantitative probe of beat-to-beat cardiovascular control. Science, 213, 220-222.

BARretT, L. F. (2004). Feelings or words? Understanding the content in self-report ratings of experienced emotion. Journal of Personality \& Social Psychology, 87, 266-281.

Barrett, L. F., \& Niedenthal, P. M. (2004). Valence focus and the perception of facial affect. Emotion, 4, 266-274.

Berntson, G. G., Bigger, T., Eckberg, D. L., Grossman, P., KaufmanN, P. G., Malik, M., ET AL. (1997). Heart rate variability: Origins, methods, and interpretive caveats. Psychophysiology, 34, 623-648.

Bradley, M. M. (2000). Emotion and motivation. In J. T. Cacioppo, L. G. Tassinary, \& G. G. Berntson (Eds.), Handbook of psychophysiology (pp. 602-642). Cambridge: Cambridge University Press.
Buchanan, T. W., Tranel, D., \& Adolphs, R. (2005). Emotional autobiographical memories in amnesic patients with medial temporal lobe damage. Journal of Neuroscience, 25, 3151-3160.

Cacioppo, J. T., Berntson, G. G., Klein, D. J., \& Poehlmann, K. M. (1997). The psychophysiology of emotion across the lifespan. Annual Review of Gerontology \& Geriatrics, 17, 27-74.

Cacioppo, J. T., Berntson, G. G., Larsen, J. T., Poehlmann, K. M., \& Ito, T. A. (2000). The psychophysiology of emotion. In M. Lewis \& J. M. Haviland-Jones (Eds.), Handbook of emotions (2nd ed., pp. 173191). New York: Guilford Press.

Cacioppo, J. T., Klein, D. J., Berntson, G. G., \& Hatfield, E. (1993). The psychophysiology of emotion. In M. B. Lewis \& J. M. Haviland (Eds.), Handbook of emotions (1st ed., pp. 119-142). New York: Guilford Press.

Carney, R. M., Freedland, K. E., Stein, P. K., Skala, J. A., HoffMAN, P., \& JAFFE, A. S. (2000). Change in heart rate and heart rate variability during treatment for depression in patients with coronary heart disease. Psychosomatic Medicine, 62, 639-647.

Carr, L., Iacoboni, M., Dubeau, M., Mazziotta, J. C., \& Lenzi, G. L. (2003). Neural mechanisms of empathy in humans: A relay from neural systems for imitation to limbic areas. Proceedings of the $\mathrm{Na}$ tional Academy of Sciences, 100, 5497-5502.

Christie, I. C., \& Friedman, B. H. (2004). Autonomic specificity of discrete emotion and dimensions of affective space: A multivariate approach. International Journal of Psychophysiology, 51, 143-153.

Dougherty, D. D., Rauch, S. L., Deckersbach, T., Marci, C. [D.], LOH, R., SHIN, L. M., ET AL. (2004). Ventromedial prefrontal cortex and amygdala dysfunction during an anger induction positron emotion tomography study in patients with major depressive disorder with anger attacks. Archives of General Psychiatry, 61, 795-804.

Ekman, P., \& Davidson, R. J. (1994). The nature of emotion: Fundamental questions. New York: Oxford University Press.

Ekman, P., Levenson, R. W., \& Friesen, W. V. (1983). Autonomic nervous system activity distinguishes among emotions. Science, 221, 1208-1210.

First, M. B., Spitzer, R. L., Gibbon, M., \& Williams, J. B. (1995). Structured clinical interview for Axis I DSM-IV disorders-patient edition (with psychotic screen) [Version 2.0]. New York: New York State Psychiatric Institute Biometrics Research Department

Friston, K. J., Frith, C. D., Liddle, P. F., \& Frackowiak, R. S. (1991) Comparing functional PET images: The assessment of significant change. Journal of Cerebral Blood Flow Metabolism, 11, 690-699.

Friston, K. J., Holmes, A. P., Worsley, K. J., Poline, J. P., Frith, C. D., \& FRACKOWIAK, R. S. (1995). Statistical parametric maps in functional imaging: A general approach. Human Brain Mapping, 2, 189-210.

Gemar, M. C., Kapur, S., Segal, Z., Brown, G. M., \& Houle, S. (1996). Effects of self-generated sad mood on regional cerebral activity: A PET study in normal subjects. Depression, 4, 81-88.

Glascher, J., \& AdolPHs, R. (2003). Processing of the arousal of subliminal and supraliminal emotional stimuli by the human amygdala. Journal of Neuroscience, 23, 10274-10282.

Goldstein, R. Z., Alia-Klein, N., Leskovjan, A. C., Fowler, J. S. WAN, G., GUR, R. C., ET AL. (2005). Anger and depression in cocaine addiction: Association with the orbitofrontal cortex. Psychiatry Research: Neuroimaging, 138, 13-22.

Hubert, W., \& De Jong-Meyer, R. (1993). Autonomic, neuroendocrine, and subjective responses to emotion inducing film stimuli. International Journal of Psychophysiology, 11, 131-140.

Hugdahl, K. (1995). Psychophysiology: The mind-body perspective. Cambridge, MA: Harvard University Press.

JAMES, W. (1884). What is an emotion? Mind, 9, 188-205.

Kawachi, I., Sparrow, D., Vokonas, P. S., \& WeIss, S. T. (1995). Decreased heart rate variability in men with phobic anxiety. American Journal of Cardiology, 75, 882-885.

Kensinger, E. A., \& Schacter, D. L. (2006). Amygdala activity is associated with the successful encoding of item, but not source information for positive and negative stimuli. Journal of Neuroscience, 26, 2564-2570.

Lang, P. J., Bradley, M. M., \& Cuthbert, B. N. (1998). International affective picture system (IAPS): Technical manual and affective ratings. Gainesville: University of Florida, Center for Research in Psychophysiology. 
Lang, P. J., Greenwald, M. K., Bradley, M. M., \& Hamm, A. O. (1993). Looking at pictures: Affective, facial, visceral, and behavioral reactions. Psychophysiology, 30, 261-273.

Murphy, F. C., Nimmo-Smith, I., \& Lawrence, A. D. (2003). Functional neuroanatomy of emotion: A meta-analysis. Cognitive, Affective, \& Behavioral Neuroscience, 3, 207-233.

OldFIELD, R. C. (1971). The assessment and analysis of handedness: The Edinburgh inventory. Neuropsychologia, 9, 97-113.

Onitsuka, T., Shenton, M. E., Salisbury, D. F., Dickey, C. C., Kasai, K., Toner, S. K., ET AL. (2004). Middle and inferior temporal gyrus gray matter volume abnormalities in chronic schizophrenia: An MRI study. American Journal of Psychiatry, 161, 1603-1611.

Phan, K. L., Wager, T., Taylor, S. F., \& Liberzon, I. (2002). Functional neuroanatomy of emotion: A meta-analysis of emotion activation studies in PET and fMRI. Neurolmage, 16, 331-348.

Pitman, R. K., Orr, S. P., Forgue, D. F., de Jong, J. B., \& Claiborn, J. M. (1987). Psychophysiologic assessment of posttraumatic stress disorder imagery in Vietnam combat veterans. Archives of General Psychiatry, 44, 970-975.
Sloan, R. P., Shapiro, P. A., Bigger, J. T., Bagiella, E., Steinman, R. C., \& Gorman, J. M. (1994). Cardiac autonomic control and hostility in healthy subjects. American Journal of Cardiology, 74, 298-300.

Velasco, C., \& Bond, A. (1998). Personal relevance is an important dimension for visceral reactivity in emotional imagery. Cognition \& Emotion, 12, 231-242.

Wager, T. D., Phan, K. L., Liberzon, I., \& Taylor, S. F. (2003). Valence, gender, and lateralization of functional brain anatomy in emotion: A meta-analysis of findings from neuroimaging. Neurolmage, 19, 513-531.

Wright, C. I., Fischer, H., Whalen, P. J., McInerney, S. C., Shin, L. M., \& RAUCH, S. L. (2000). Differential prefrontal cortex and amygdala habituation to repeatedly presented emotional stimuli. NeuroReport, 12, 379-383.

(Manuscript received July 9, 2006;

revision accepted for publication March 10, 2007.) 